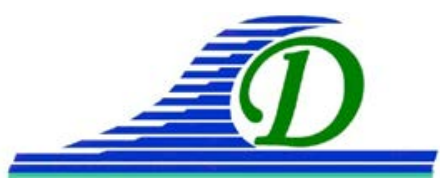

$$
\begin{gathered}
\text { XIII }^{\text {èmes }} \text { Journées Nationales Génie Côtier - Génie Civil } \\
\text { Dunkerque, 2-4 juillet } 2014
\end{gathered}
$$

DOI:10.5150/jngcgc.2014.112 C Editions Paralia CFL

disponible en ligne - http://www.paralia.fr - available online

\title{
Effets des cendres volantes sur le comportement mécanique de sédiments méditerranéens traités à base de ciment et de chaux
}

\author{
Yingjie LIANG ${ }^{1,2}$, Daniel LEVACHER ${ }^{2}$, Dimitri DENEELE ${ }^{1,3}$, \\ Andry RAZAKAMANANTSOA ${ }^{1}$
}

1. LUNAM, IFSTTAR, Institut Français des Sciences et des Technologies des Transports, de l’Aménagement et des Réseaux, BP 4129, route de Bouaye, 44332 Bouguenais, France.

2. Université de Normandie, Unicaen, GRGCE, UMR 6143 CNRS - M2C, Caen, 24 rue des Tilleuls, 14000 Caen, France.

3. Institut des Matériaux Jean Rouxel (IMN), Université de Nantes, CNRS, 2 rue de la Houssinière, BP 32229, 44322 Nantes Cedex 3, France.

yingjie.liang@ifsttar.fr ; daniel.levacher@unicaen.fr

\section{Résumé :}

Des sédiments de dragage méditerranéens provenant de six ports ont été stabilisés voire solidifiés avec des liants hydrauliques classiques : ciment et chaux. Une incorporation de 10\% de cendres volantes (co-produits industriels de type F) a été menée sur des mélanges sédiments-liants dont les dosages de base étaient $7 \%$ de ciment et $2 \%$ de chaux. Cette incorporation de cendres volantes permet une amélioration du comportement mécanique validée par des essais de résistance en compression simple $R c$ - effectués aux dates conventionnelles de 14, 28 et 60 jours.

Les cendres volantes dont la teneur élevée en éléments $\mathrm{Si}$ et $\mathrm{Al}$, peuvent contribuer à accélérer la réaction d’hydratation. On a bien observé que les valeurs de $R c$ à la rupture des sédiments traités avec $10 \%$ de cendres volantes augmentent plus rapidement jusqu'à 28 jours par rapport à celles obtenues pour les sédiments sans addition de cendre volantes. Au-delà de 28 jours, on note une diminution des valeurs de Rc acquises pour les sédiments traités avec $10 \%$ de cendres volantes. Considérant d'une part, les principales caractéristiques physico-chimiques des sédiments méditerranéens et liants étudiés et d'autre part les conditions de cure, les effets des cendres volantes incorporées dans les mélanges sédiments-liants de base, sont analysés. Des explications sont apportées à ces observations.

Mots-clés : Sédiments, Cendres volantes, Résistance à la compression, Caractéristiques physico-chimiques, Condition de cure, Hydratation, Chaux, Effet pouzolanique.

\section{Introduction}

De récentes études ont montré une nette tendance à la diversification des voies de valorisation pour les sédiments de dragage en génie civil. Les applications concernent 
les composés cimentaires, les bétons et mortiers, les matériaux routiers et de remblai, les céramiques (LIMEIRA et al., 2010 ; AOUAD et al., 2012 ; DANG et al., 2013 ; DALTON et al., 2004 ; GARDNER et al., 2007 ; ZENTAR et al., 2008 ; ANGER et al., 2013). Cependant la filière matériau routier, de par les volumes de sédiments susceptibles d'être traités reste la plus étudiée. Des mélanges sédiments fins-liants peuvent constituer une voie de valorisation pour des sous-couches routières dans la mesure où les teneurs en matières organiques et en métaux lourds dans ces sédiments sont acceptables (COLIN, 2003 ; DUAN, 2008 ; SILITONGA, 2010 ; LIANG, 2012). Simple dans sa mise en œuvre, cette technique de stabilisation/solidification aux liants hydrauliques s'avère tout aussi intéressante en intégrant les critères environnementaux et économiques (SILITONGA, 2010; KAMALI et al., 2008; TRIBOUT, 2010 ; MIRAOUI et al., 2012 ; SANNIER et al., 2008). La chaux modifie les limites d'Atterberg (LEROUEIL \& LE BIHAN, 1996), la structure et les propriétés d'un sol fin (RAZAKAMANANTSOA et al., 2012 ; CAI et al., 2006), fait augmenter le pH (LIONS, 2004) et conduit à modifier les propriétés macroscopiques par réaction pouzzolanique entre les minéraux du sol (POMAKHINA et al., 2012). La chaux peut aussi empêcher la matière organique d'inhiber la réaction d'hydrolyse du ciment (TREMBLAY et al., 2002). Des cendres volantes peuvent être ajoutées à des mélanges à base de chaux pour en améliorer les performances mécaniques à court terme selon CROISE (1964). Elles peuvent aussi controler le gonflement (BIN-SHAFIQUE et al., 2010).

L'objectif de cette étude est d'évaluer l'apport de chaux, de ciment et de cendres volantes dans la stabilisation des sédiments portuaires méditerranéens, déssablés et déshydratés (DD). Une déshydratation et un tri granulométrique ont été initialement opérés sur les sédiments bruts. Ces prétraitements constituent un préalable à toute valorisation matière. L'influence de la granulométrie, de l'argilosité, de la matière organique et des carbonates des sédiments traités aux liants hydrauliques et aux cendres volantes sur les résistances mécaniques est analysée. Puis le rôle de la cendre volante dans les mélanges sédiments-liants est expliqué à partir de l'évolution des paramètres de résistance mécanique et des conditions de cure.

\section{Matériel et méthodes}

\subsection{Sédiments Méditerranéens étudiés}

Six échantillons dessablés/déshydratés (DD) correspondent aux sédiments prélevés dans les ports méditerranéens suivants : Arsenal de Toulon (ARS), Ports de Bandol (BAN), de Cannes (CAN), de Sanary-Lavandou (SAN), de Saint-Mandrier (STM) et le Vieux Port de Marseille (VPM). Leurs principales caractéristiques physico-chimiques en tant que sédiments prétraités DD sont données au tableau 1. 


\section{XIII ${ }^{\text {èmes }}$ Journées Nationales Génie Côtier - Génie Civil \\ Dunkerque, 2-4 juillet 2014}

Tableau 1. Propriétés physico-chimiques des sédiments Méditerranéens étudiés.

\begin{tabular}{|c|c|c|c|c|c|c|}
\hline \multirow[t]{2}{*}{ Caractérisation } & \multicolumn{6}{|c|}{ Catégorie de sédiments } \\
\hline & $A R S / D D$ & $B A N / D D$ & $C A N / D D$ & $S A N / D D$ & $S T M / D D$ & $V P M / D D$ \\
\hline \multicolumn{7}{|l|}{ Répartition des grains } \\
\hline Fraction sableuse (> $2 \mathrm{~mm}$ ), (\%) & 0.2 & 1.5 & 0 & 0 & 0 & 0 \\
\hline Fraction sableuse (63 $\mu \mathrm{m}$ à $2 \mathrm{~mm}$ ), (\%) & 24.8 & 23.6 & 30.9 & 37.3 & 26.4 & 25.6 \\
\hline 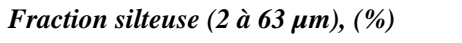 & 66.3 & 64.1 & 48.3 & 53.2 & 64.7 & 65.2 \\
\hline Fraction argileuse (< $2 \mu \mathrm{m}),(\%)$ & 8.7 & 10.8 & 20.8 & 9.5 & 8.9 & 9.2 \\
\hline Coefficient d'uniformité $\mathrm{Cu}$ & 13.6 & 21.8 & 60.6 & 26.2 & 16.4 & 15 \\
\hline Coefficient de courbure Cc & 1.0 & 1.3 & 1.0 & 1.3 & 1.2 & 1.1 \\
\hline \multicolumn{7}{|l|}{ Limites d'Atterberg } \\
\hline Limite de liquidité (\%) & 77 & 71 & 72 & 97 & 118 & 87 \\
\hline Limite de plasticité (\%) & 59 & 55 & 53 & 58 & 94 & 73 \\
\hline Indice de plasticité (\%) & 18 & 16 & 19 & 39 & 24 & 14 \\
\hline \multicolumn{7}{|l|}{ Autres constituants } \\
\hline Teneur en eau initiale (\%) & 44 & 42 & 43 & 52 & 89 & 51 \\
\hline Matière organique (\%) & 20.5 & 13.1 & 14.8 & 18.5 & 24.1 & 20 \\
\hline Carbonates $\left(\mathrm{CaCO}_{3}\right)(\%)$ & 16.8 & 25.9 & 21.6 & 11 & 13.6 & 13.6 \\
\hline
\end{tabular}

Ce sont des sédiments limoneux, plastiques à peu plastiques, de taille $0 / 2 \mathrm{~mm}$. La teneur en matière organique et en carbonates varie respectivement de 13 à $25 \%$ et de 11 à $26 \%$ en fonction des différents sites de prélèvement.

\subsection{Liants hydrauliques et cendres volantes}

Le type de ciment choisi pour les mélanges est un ciment CEM II B-M (S-LL) 32,5R CE CP1 NF. La chaux utilisée est une chaux de marque Dugay, une chaux vive en poudre 0/1.5 mm, de teneur en $\mathrm{CaO}$ supérieure à 93\%. Pour les mélanges étudiés avec ces liants, une cendre volante est ajoutée. La cendre volante (CV) retenue, la Sodeline ${ }^{\circledR}$, est de nature silico-alumineuse (tableau 2). Elle a une structure très poreuse et se présente sous la forme d'une poudre très fine et légère, composée d'un assemblage de plaquettes et de fragments alvéolaires.

Tableau 2. Composition chimique de la cendre volante Sodeline ${ }^{\circledR}$.

\begin{tabular}{lllllllllll}
$(\%)$ & $\mathbf{S i O}_{2}$ & $\mathrm{Fe}_{2} \mathrm{O}_{3}$ & $\mathrm{Al}_{2} \mathrm{O}_{3}$ & $\mathbf{M g O}$ & $\mathbf{M n O}_{2}$ & $\mathbf{C a O}_{\text {total }}$ & $\mathrm{CaO}_{\text {libre }}$ & $\mathrm{Na}_{2} \mathrm{O}$ & $\mathrm{K}_{2} \mathrm{O}$ & $\mathbf{S O}_{3}$ \\
\hline Sodeline $^{\circledast}$ & 47.36 & 7.09 & 21.63 & 3.32 & 0.62 & 8.52 & 0.90 & 0.46 & 4.35 & 4.02 \\
\hline
\end{tabular}

\subsection{Préparation, conservation et protocoles}

Deux formulations économiques de mélanges ont été proposées dans cette étude, avec ajout ou non de cendres volantes (tableau 3). Les 6 sédiments ont été séchés dans une étuve à $60{ }^{\circ} \mathrm{C}$. Les éprouvettes sont confectionnées par compactage manuel dans des moules cylindriques de diamètre $34 \mathrm{~mm}$ avec un élancement de 2. Puis elles sont 
démoulées et conservées à l'air ambiant à une température de $20^{\circ} \mathrm{C} \pm 2{ }^{\circ} \mathrm{C}$. Les conditions de cure ont été préconisées en fonction des applications prévues. Les performances mécaniques de résistance sont déterminées à partir d'essais de résistance à la compression simple $R c$ à $j$ jours (avec $j=14,28$ et 60 jours) effectués.

Tableau 3. Composition des mélanges sédiments/liants étudiés.

\begin{tabular}{llllll}
\hline Echantillons & Eau (\%) & Ciment (\%) & Chaux (\%) & Sodeline $^{\circledR}$ & Mélange \\
\hline Sédiments/DD & 50 & 7 & 2 & - & Série A \\
Sédiments/DD & 50 & 7 & 2 & 10 & Série B \\
\hline
\end{tabular}

\section{Résultats et discussion}

\subsection{Analyse de la résistance des mélanges sédiments-ciment-chaux}

Les mélanges de la série A portent sur un traitement de base aux liants hydrauliques classiques : $7 \%$ de ciment et $2 \%$ de chaux. L'évolution des résistances à la compression obtenues aux dates de 14, 28 et 60 jours est montrée sur la figure 1a. Les valeurs observées de $R c$ des sédiments ARS, BAN, CAN et SAN sont les plus élevées et supérieures à $1 \mathrm{MPa}$ à 14, 28 et 60 jours. La figure 1 montre, que pour les sédiments STM et VPM, les valeurs de $R c$ apparaissent comme les plus faibles et en deçà du seuil de trafficabilité de 1 MPa requis par le GTS (2000). Même si on note dans le cas des sédiments STM des valeurs respectives de $R c$ de $1.38 \mathrm{MPa}$ et $1.29 \mathrm{MPa}$ à 14 et 28 jours ; la valeur à 60 jours n'est plus que de $0.98 \mathrm{MPa}$. Le comportement mécanique de ces deux sédiments VPM et STM diffère des 4 autres. Par ailleurs on n’observe pas de stabilisation de la résistance à 60 jours pour la moitié des sédiments étudiés.

\subsection{Analyse de la résistance des mélanges sédiments-ciment-chaux-cendres volantes}

La figure $1 \mathrm{~b}$ montre l'évolution des résistances obtenues pour les sédiments/DD traités avec du ciment, de la chaux et des cendres volantes. On note une nette amélioration de l'ensemble des valeurs de Rc. En effet, pour les dosages de ces mélanges correspondant à $7 \%$ de ciment, $2 \%$ de chaux et $10 \%$ de cendres volantes, les valeurs obtenues de $R c$ sont plus élevées que celles de la série $\mathrm{A}$ et toutes sont égales ou supérieures à $1 \mathrm{MPa}$ à 28 jours. Quelle que soit la série de mélanges, on note que les valeurs de $R c$ des sédiments BAN et VPM diminuent toujours avec le temps de cure. Les sédiments VPM présentent même une résistance inférieure à $1 \mathrm{MPa}$ à 60 jours. Ceci pourrait s'expliquer par leurs caractéristiques physico-chimiques qui viendraient perturber l'hydratation de ciment (TAYLOR, 1997 ; HEWLETT, 1998) et par voie de conséquence, diminuer leur performance mécanique durant le temps de cure. 


\section{XIII ${ }^{\text {èmes }}$ Journées Nationales Génie Côtier - Génie Civil \\ Dunkerque, 2-4 juillet 2014}
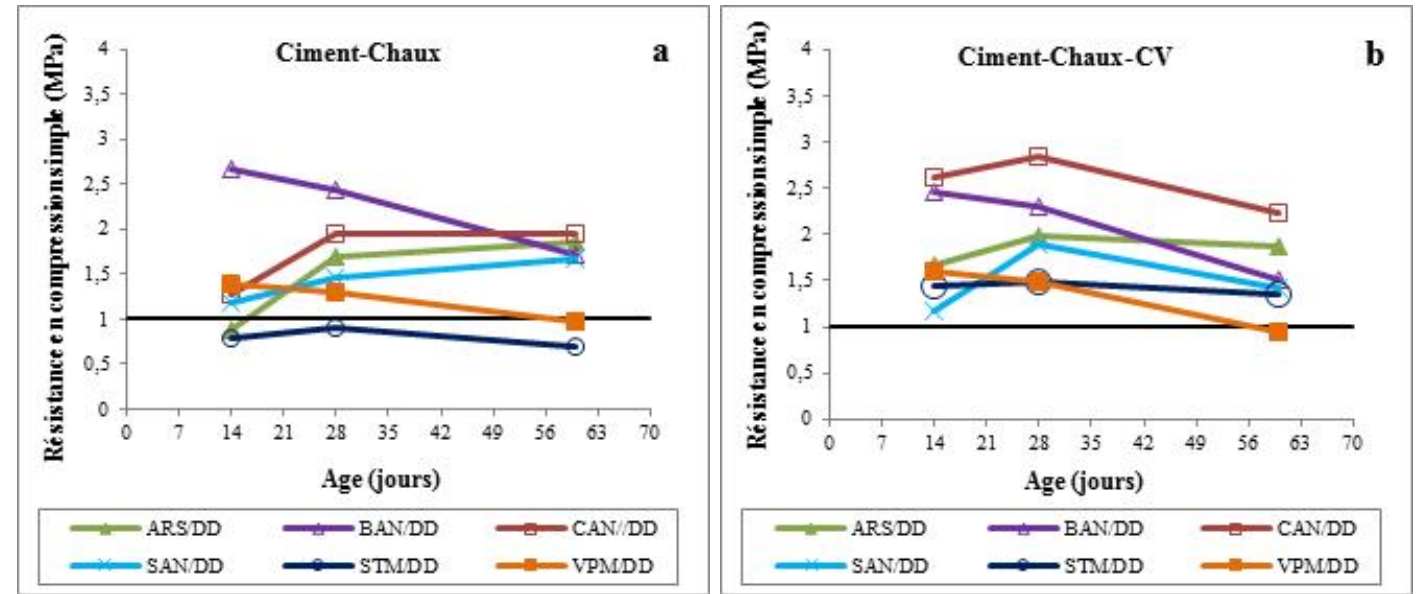

Figure 1. Evolution comparée des résistances à la compression pour les formulations ciment-chaux-cendres volantes des sédiments déshydratés-dessablés Méditerranéens (a) formulation à base de chaux et de ciment ; (b) formulation à base de chaux, de ciment et de cendre volante.

\subsection{Effet de la cendre volante sur la stabilisation des mélanges étudiés}

Afin de montrer l'effet de la cendre volante sur le comportement mécanique, la figure 2 montre le rapport des valeurs de $R c$ obtenues pour les mélanges ciment-chaux et cimentchaux-CV à 14, 28 et 60 jours. On observe à 14 jours que les valeurs de $R c$ pour 3 sédiments (ARS, CAN et STM) sont nettement plus élevées avec un ajout de cendres volantes que celles obtenues sans ajout de CV, et ce, jusqu'à 28 jours. Le taux d'augmentation reste variable selon les sédiments, mais l'effet de l'ajout est observé excepté le sédiment BAN comme montré sur les figures $1 \mathrm{~b}$ et 2 . Cependant à long terme (à 60 jours), l'effet semble s'estomper sauf pour le sédiment STM. C'est-à-dire que les valeurs de Rc des mélanges ciment-chaux-CV fluctuent avec le temps de cure.

Si l'on considère la teneur élevée en $\mathrm{Si}$ et $\mathrm{Al}$ (tableau 2) de la cendre volante, son incorporation dans le mélange accélére l'hydratation des $\mathrm{C}_{3} \mathrm{~A}$ et $\mathrm{C}_{3} \mathrm{~S}$ du ciment (HEWLETT, 1998). Les sédiments CAN et STM avec 10\% de CV, fournissent des valeurs moyennes à 28 jours plus beaucoup élevées que celles obtenues sans cendres. Cependant, après 28 jours, les valeurs des $R c$ tendent à diminuer plus ou moins vite. Il y a une difficulté à stabiliser les valeurs de $R c$ observées.

Par ailleurs, si l'on considère les sédiments CAN et STM, on observe que les cendres volantes ajoutées peuvent contribuer à l'amélioration des résistances Rc pour ces sédiments dont la teneur en argile et/ou en matière organique est élevée. Pour autant, les valeurs de $R c$ des sédiments avec $\mathrm{CV}$ ne sont pas stabilisées à 60 jours, probablement en réponse à l'évolution physico-chimique du matériau (hydratation du ciment et la réaction pouzzolanique entre la cendre volante et la chaux). 


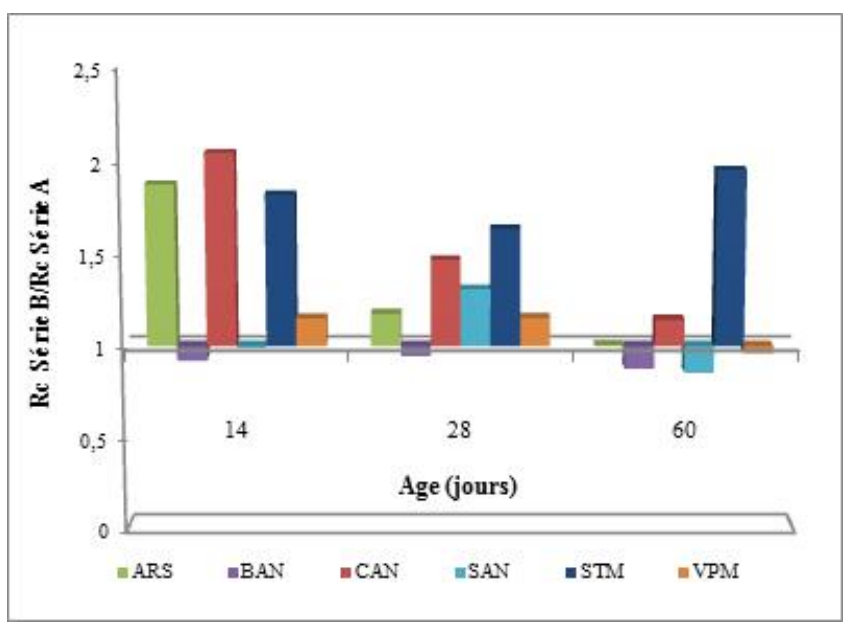

Figure 2. Evolution du rapport $R c_{\text {sérieB }} / R c_{\text {sérieA }}$ en fonction du temps de cure.

3.4 Effet de la teneur en matière organique sur la stabilisation des sédiments par liants La teneur en matière organique M.O. est un paramètre important dans toute valorisation matériau avec des liants (TREMBLAY et al., 2002). L’effet de la M.O. est très marqué, et de manière continue, avec les sédiments déshydratés/dessablés quel que soient les mélanges étudiés: ciment-chaux et ciment-chaux-CV, voir figure 3. Il y a une décroissance de $R c$ au fur et à mesure que la teneur en matière organique augmente. Par ailleurs, on note que pour un taux en matière organique supérieur à $20 \%$, ce qui est le cas des sédiments STM et VPM, le seuil de 1 MPa serait difficile à atteindre pour les mélanges sédiments + ciment et chaux. Pour la résistance en compression des sédiments avec un ajout de CV, la même tendance est observée. L’ajout de 10\% de CV atténue l'effet de la teneur en M.O. (figure 3).

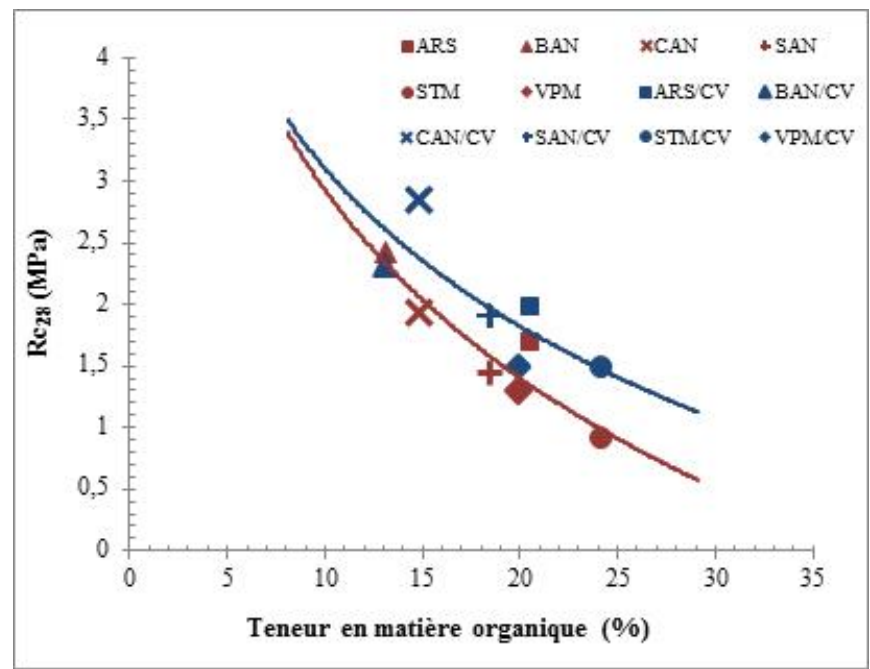

Figure 3. Relation Rc28 - matières organiques pour les formulations étudiées. 


\section{XIII ${ }^{\text {èmes }}$ Journées Nationales Génie Côtier - Génie Civil \\ Dunkerque, 2-4 juillet 2014}

3.5 Effet de la teneur en carbonates sur la stabilisation des sédiments par liants

La teneur en carbonates de calcium est un paramétre intéressant pour la stabilisation du sol (RAMESH et al., 2012 ; PARK et al., 2014). La relation entre les résistances et cette teneur est illustrée sur la figure 4. Pour le mélange à base de de ciment-chaux, il y une nette tendance à l'augmentation des valeurs de $R c$ en fonction de la teneur en carbonates. Pour les sédiments des mélanges ciment-chaux-CV, la tendance croissante est toujours observée même si elle est moins marquée. L'addition de $10 \%$ de CV diminue l'effet sur le comportment mécanique de la teneur en carbonates.

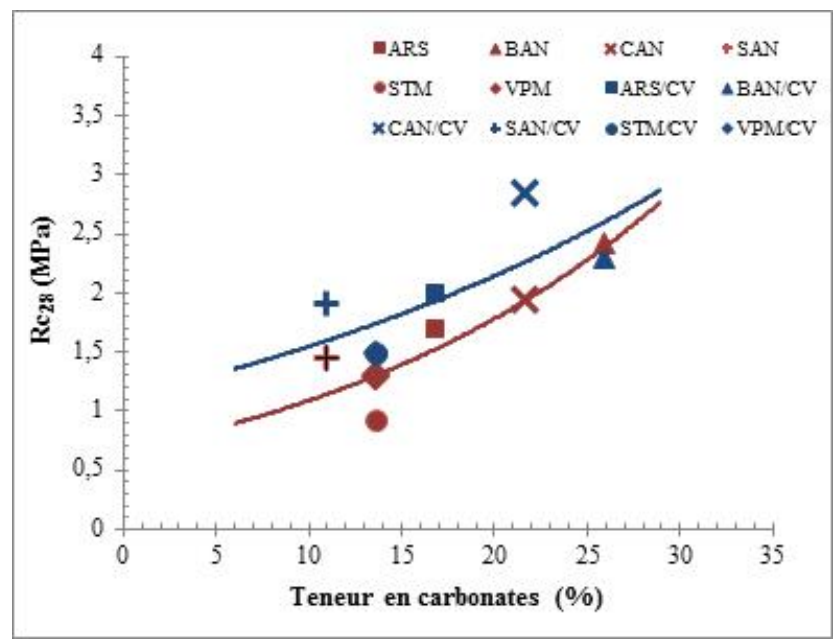

Figure 4. Relation Rc28 - carbonates pour les formulations étudiées.

\section{Conclusions}

Les 2 formulations des mélanges étudiées, à base de $7 \%$ de ciment et 2\% de chaux avec ou sans $10 \%$ de cendres volantes ont permis :

- de stabiliser les sédiments et d'acquérir une valeur suffisante de résistance à la compression proche de $1 \mathrm{MPa}$,

- de constater que les valeurs de Rc sont plus stables de 28 à 60 jours,

- d'observer que $10 \%$ de cendres volantes mèneraient à une dimunution de Rc entre 28 à 60 jours même si les valeurs de $R c$ obtenues sont plus élevées que celles des sédiments de ciment-chaux entre 14 et 28 jours,

- d'étudier l'influence de la teneur en matière organique. En quantité importante elle influe sur le comportement mécanique des sédiments marins stabilisés aux liants. Ceci a été constaté pour les deux mélanges étudiés. Un ajout de cendres volantes à un traitement à base de ciment et de chaux peut améliorer les performances mécaniques à court terme ( $<28$ jours) sauf pour les sédiments à teneur élevée en argile et/ou en matière organique. On observe aussi, un fléchissement des valeurs de $R c$ au-delà de 28 jours pour tous les mélanges à base de cendre volante, 
- de mettre en evidence l'influence de la teneur en carbonates des sédiments initiaux sur leur performance mécanique. Une analyse plus fines de la microstructure est nécessaire pour mieux comprendre le mécanisme des réactions mis en jeux.

\section{Références}

ANGER B., LECOMTE-NANA G., PEYRATOUT C., THERY B., LEVACHER D. (2013). Les retenues hydroélectriques : source renouvelable de matières premières pour les céramiques silicatées ? L’Industrie Céramique et Verrière, $\mathrm{n}^{\circ} 1047$, pp 40-45.

AOUAD G., LABOUDIGUE A., GINEYS N., ABRIAK N.E. (2012). Dredged sediments used as novel supply of raw material to produce Portland cement clinker. Cement and Concrete Composites, Vol. 34(6), pp 788-793. http://dx.doi.org/10.1016/j.cemconcomp.2012.02.008

BIN-SHAFIQUE S., RAHMAN K., YAYKIRAN M., AZFAR I. (2010). The long-term performance of two fly ash stabilized fine-grained soil subbases. Resources, Conservation and Recycling, Vol. 54(10), pp 666-672. http://dx.doi.org/10.1016/j.resconrec.2009.11.007

CAI Y., SHI B., NG C. W. W., TANG C. (2006). Effect of polypropylene fibre and lime admixture on engineering properties of clayey soil. Engineering Geology, Vol. 87(3-4), pp 230-240. http://dx.doi.org/10.1016/j.enggeo.2006.07.007

COLIN D. (2003). Valorisation de sédiments fins de dragage en technique routière. Thèse de doctorat, Université de Caen, 181 p.

CROISE R. (1964). Traitement des sols aux liants hydrauliques. Ecole Nationale du Génie Rural, des Eaux et des Forêts, Nancy, pp 427-438.

DALTON J.L., GARDNER K.H., SEAGER T.P., WEIMER M.L., SPEAR J.C.M., MAGEE B.J. (2004). Properties of Portland cement made from contaminated sediments. Resources, Conservation and Recycling, Vol. 41(3), pp 227-241. http://dx.doi.org/10.1016/j.resconrec.2003.10.003

DANG T.A., KAMALI-BERNARD S., PRINCE W.A. (2013). Design of new blended cement based on marine dredged sediment. Construction and Building Materials, Vol. 41, pp 602-611. http://dx.doi.org/10.1016/j.conbuildmat.2012.11.088

DUAN Z. (2008). Caractérisation, stabilisation et solidification de sédiments fins marins. Thèse de doctorat, Université de Caen, $154 \mathrm{p}$.

GARDNER K.H., TSIATSIOS C.J., MELTON J., SEAGER T. (2007). Leaching behavior of estuarine sediments and cement-stabilized sediments in upland management environments. Vol. 27, pp 1648-1654.

GTS (2000) Guide technique du Traitement des sols à la chaux et/ou aux liants hydraulique, GTS SETRA LCPC, janvier 2000, 240p.

KAMALI S., BERNARD F., ABRIAK N. E., DEGRUGILLIERS P. (2008). Marine dredged sediments as new materials resource for road construction. Waste Management, Vol. 28(5), pp 919-28. http://dx.doi.org/10.1016/j.wasman.2007.03.027 


\section{XIII ${ }^{\text {èmes }}$ Journées Nationales Génie Côtier - Génie Civil \\ Dunkerque, 2-4 juillet 2014}

HEWLETT P. (1998). Lea's Chemistry of Cement and Concrete (4th ed., p. 1053). John Wiley \& Sons, England.

LEROUEIL S., LE BIHAN J.-P. (1996). Liquid limits and falling cones. Canadian Geotechnical Journal, Vol. 33, pp 793-798. http://dx.doi.org/10.1139/t96-104-324

LIANG Y. (2012). Co-valorisation de sédiments et de sols fins par apport de liants et de fibres. Thèse de doctorat, Université de Caen, France, 194 p.

LIONS J. (2004). Etude hydrogéochimique de la mobilité de polluants inorganiques dans des sédiments de curage mis en dépôt : expérimentations, études in situ et modélisation. Thèse de doctorat, Ecole Nationale Supérieure des Mine de Paris. France, 248 p.

LIMEIRA J., AGULLO L., ETXEBERRIA M. (2010). Dredged marine sand in concrete: An experimental section of a harbor pavement. Construction and Building Materials, Vol. 24(6), pp 863-870. http://dx.doi.org/10.1016/j.conbuildmat.2009.12.011

MIRAOUI M., ZENTAR R., ABRIAK N.E. (2012). Road material basis in dredged sediment and basic oxygen furnace steel slag. Construction and Building Materials, Vol. 30, pp 309-319. http://dx.doi.org/10.1016/j.conbuildmat.2011.11.032

PARK K., JUN S., KIM D. (2014). Effect of Strength Enhancement of Soil Treated with Environment-Friendly Calcium Carbonate Powder. Hindawi Publishing Corporation The Scientific World Journal, 2014(Kctc 3558), pp 1-12.

POMAKHINA E., DENEELE D., GAILLOT A.-C., PARIS M., OUVRARD G. (2012). 29Si solid state NMR investigation of pozzolanic reaction occurring in lime-treated Cabentonite. Cement and Concrete Research, Vol. 42(4), pp 626-632. http://dx.doi.org/10.1016/j.cemconres.2012.01.008

RAMESH P., NARASIMBA RAO A.V., KRISHNA MURTHY N. (2012). Efficacy of sodium carbonate and calcium carbonate in stabilizing a black cotton soil. Engineering, Civil Vidyanikethan, Sree College, Engineering, Vol. 2(10), pp 197-201.

RAZAKAMANANTSOA A., DAVID J.P., RAYSSAC E. (2012). Effect of the sample preparation on geotechnical properties of partially saturated lime treated silt. $3^{\text {rd }}$ International Seminar on Earthworks in Europe, Berlin, 19-20 March 2012.

SANNIER L., LEVACHER D., JOURDAN M. (2008). Discrimination économique de méthodes de traitements de sédiments marins contaminés à l'aide de liants hydrauliques. $\mathrm{X}^{\text {èmes }}$ Journées Nationales Génie Côtier-Génie Civile, Sophia Antipolis, pp 821-830. http://dx.doi.org/10.5150/jngcgc.2008.077-S

SILITONGA E. (2010). Valorisation des sédiments marins contaminés par solidification / stabilisation à base de liants hydrauliques et de fumée de silice. Thèse de Doctorat, Université de Caen, 227p.

TAYLOR H.F.W. (1997). Cement chemistry. 2nd edition (p. 459). Thomas Telford Services Ltd, 1 Heron Quay, London E144JD. 
TREMBLAY H., LEROUEIL S., LOCAT J. (2002). Influence of the nature of organic matter on the soil stablization with cement. Canadian Geotechnical Journal, Vol. 39, pp 535-546. http://dx.doi.org/10.1139/t02-002

TRIBOUT C. (2010). Valorisation de sédiments traités en techniques routières : contribution à la mise en place d'un protocole d'acceptabilité. Thèse de doctorat, Université de Toulouse, 295 p.

ZENTAR R., DUBOIS V., ABRIAK N.E. (2008). Mechanical behaviour and environmental impacts of a test road built with marine dredged sediments. Resources, Conservation and Recycling, Vol. 52(6), pp 947-954. http://dx.doi.org/10.1016/j.resconrec.2008.02.002 